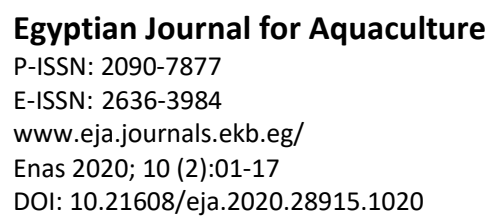

\title{
Pollutants impacts on some Chemical Characteristic of Lake Mariout sediment - Egypt
}

\section{Enas M.G. Mansour}

Central Lab. For Aquaculture Research, Abbassa, Abou - Hammad, Sharkia, Agricultural research center, Egypt, Limnology department

Received: April. 28, 2020; Accepted: May. 19, 2020 published: Vol.10 (2):01-17; 2020

\begin{abstract}
The Mariout Lake is considered an important natural resource for fish production in Alexandria Governorate, it has been subjected to various types of pollution, the lake acts as a receptor for domestic wastewater and industrial wastes. So this study was conducted to investigate the effect of environmental impacts and pollutants on some chemical properties of different cores of Mariout lake sediment, Surficial $(0-10 \mathrm{~cm})$ and sub-surficial $(10-20 \mathrm{~cm})$ sediments were collected seasonally using vertical soilsampler from different seven sites during winter, spring, summer, and autumn, 2017. The study showed that the mean values of organic matter, organic carbon total carbon, total nitrogen and total phosphorus were significantly $(\mathrm{P}<0.05)$ increased at site 3 . Obtained results showed that the different sites and different cores had been affected the investigated chemical properties of the sediment where all theses parameters decreased with the depth. OSI values ranged from (0.41to 0.87), which indicated that the degree of pollution at all sites was considered slight pollution except for sewage site which was described as high pollution. It's concluded that the Mariout Lake is subjected to different types of pollution and organic matter is mainly of exogenous organic matter. And the control of sediment nutrient concentrations depends upon the control of exogenous pollution.
\end{abstract}

Key words: Mariout lake, chemical properties, pollution, seasons

\section{Introduction}

Sediments are composite materials, consisting of inorganic components, mineral particulates, and organic matter in various stages of 
decomposition. They are sensitive and useful indicators of changes due to natural and anthropogenic events (Kucuksezgin et al. 2006). In addition, sediment quality is a good indicator of pollution in water column, where it tends to concentrate organic pollutants. Sediments show strong tendency to accumulate contaminant. Knowledge of sedimentology and chemistry of the sediment is a key condition for identification of their sources and assessment of their transport-dispersion patterns. Furthermore, they have high physical-chemical stability, and their characteristics usually represent the average condition of the system (Leoni and Sartori 1996). Thus chemical characteristics measured in this investigation gave descriptive criteria on the nature of Mariout sediment.

Lake Mariout, for a long time, represents a source of fish production in Egypt. Currently, some parts of the lake are used in aquaculture activities. It is one of the most heavily populated urban areas in Egypt (Mateo 2009; Saad et al. 2017). The lake has been subjected to various anthropogenic activities. The lake acts as a receptor for agricultural drainage and domestic wastewater. The main drains and canal are Qalaa drain, El-Umum drain, and Nubaria canal. The water inflow to the lake comes mainly from these sources plus those from the East and West treatment plants began in 1993 to treat Alexandrian waste water. Both are primarily treatment plants and they discharge their final effluents into the lake and from the petrochemical area, domestic sewage from the Wastewater Treatment Plant at the north and El-Qalaa drain are discharging in the main basin of the lake. Current water characteristics of the lake were not suitable for aquaculture activities where the basin receives daily about $200,000-300,000 \mathrm{~m}^{3}$ /day of sewage and $400,000 \mathrm{~m}^{3} /$ day of agricultural waste water mixed with sewage. In addition discharging about 25,000-35,000 m3/day of raw sewage and some industrial waste water into the lake (Scapini, 2009). Throughout the last four decades, the lake has been subjected to man-made changes in its morphometric features as its area has been reduced. Lake Mariout is highly polluted. It receives the drastic human impacts among the Egyptian lakes. It has been greatly deteriorated from a productive lake to a heavily polluted and highly eutrophicated basin (Gehan et al., 2018).

\section{Material and methods}

\section{Study area}

Lake Mariout is one of the main fishing grounds of Alexandria located between longitude $29^{\circ} 47.1^{\prime}$ to $29^{\circ} 50.4^{\prime} \mathrm{E}$ and latitude $31^{\circ} 7.5^{\prime}$ to $31^{\circ} 9^{\prime} \mathrm{N}$ (Saad et al., 2017). The Lake is a shallow brackish water basin and its area is less than $65 \mathrm{~km}^{2}$, its water depth ranges from 1 to $3 \mathrm{~m}$. It lies at the north of the Nile Delta, southeast of Alexandria. In recent years, Lake Mariout 
was considered as an oxidation pond for Alexandrian waste water and agricultural wastes, which discharged to it by the newly constructed plant stations. To evaluate the sediment quality, the samples were collected from the following sites, namely, Resha, petrochemical, sewage drain, 1000 feddan (fish basin), 2000 feddan (central basin), 3000 feddan (west basin), and 6000 feddan basin (Main basin) as shown in (Figure.1)

\section{Sampling}

Surficial $(0-10 \mathrm{~cm})$ and sub-surficial $(10-20 \mathrm{~cm})$ sediments were collected seasonally using vertical soilsampler from seven sites during winter, spring, summer, and autumn 2017 and stored in pre-cleaned aluminum containers for analysis. Samples of sediments were air dried and subjected to grain size analysis.

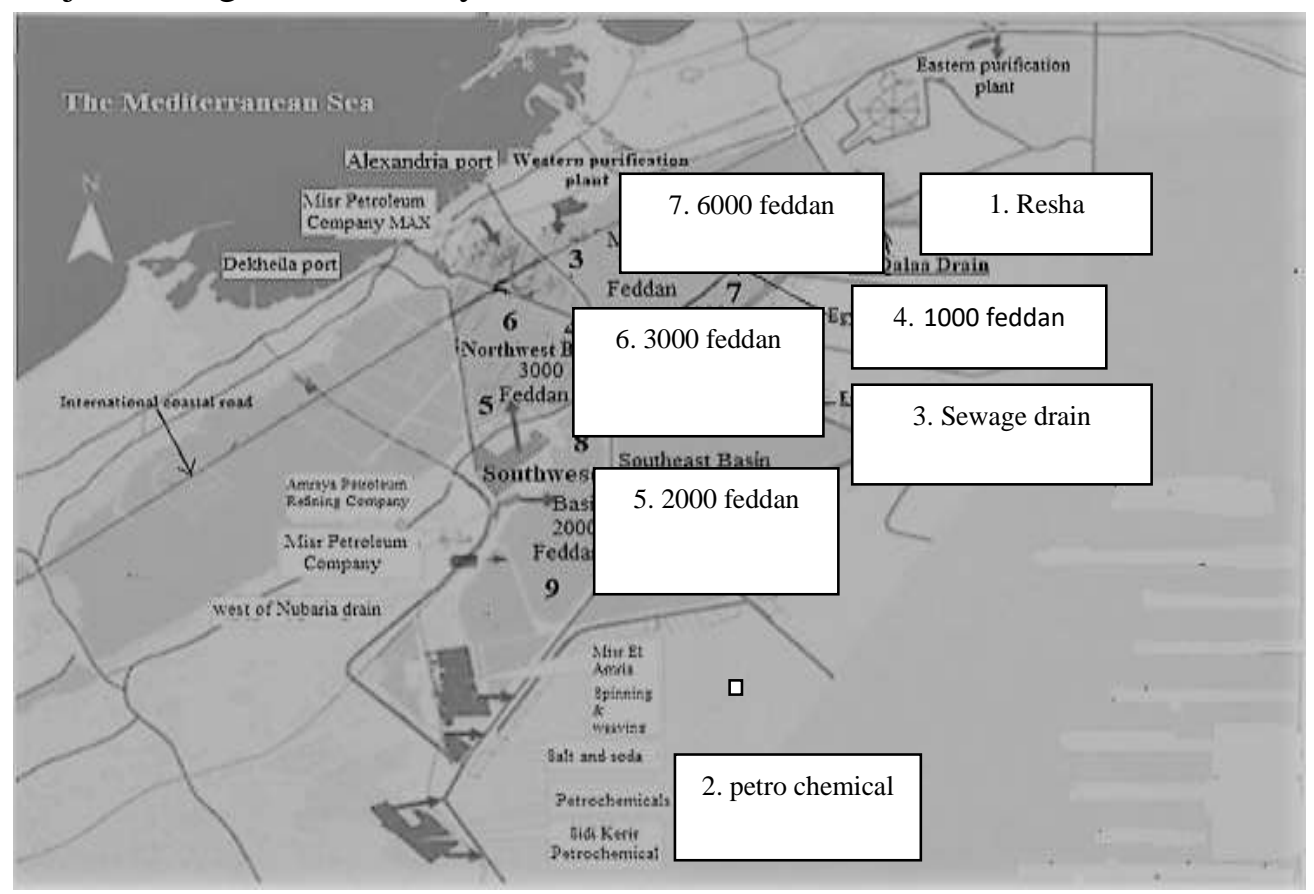

Figure (1). Show the mains pond of Lake Mariout and seven sites.

\section{Sediment analysis}

Soil $\mathrm{pH}$ was measured with digital $\mathrm{pH}$ meter (Model 25, Fisher Scientific) which its electrode inserted into a 1:1 mixture of dry, pulverized soil and distilled water. Organic matter was measured as loss in weight after ignition at $550^{\circ} \mathrm{C}$ for $3 \mathrm{~h}$ (Page et al., 1982), while organic carbon (OC \%) was calculated using the conventional conversion: $\mathrm{OM}=1.72 \times \mathrm{OC}$ (Boyd, 1995). Total phosphorus (TP) was measured using the dry ash method (Tavares and Boyd, 2003) for digestion then phosphorus was 
colorimetrically estimated using the vanadomolybdate method (APHA, 1985). Total nitrogen content was estimated by using Kjeldalh method (A.O.A.C. 1990).

\section{Assessment of Organic Sediment Index}

The organic load was evaluated by the Organic Sediment Index (OSI) of Ballinger and McKee (1971) as follow: OSI $=(\mathrm{OC} w \mathrm{wt} \%) \times(\mathrm{ON} w \mathrm{wt})$, where (OC wt \%) was defined as the ratio between the organic matter $(\%)$ and 1.724 , while (ON wt $\%$ ) was the value of total nitrogen multiplied by 0.95 . The OSI had a potential range of minus ten $(-10)$ to plus eleven $(+11)$ which reflected the ecological status (in terms of organic loading) of a particular survey and/or station. This index was developed for the classification of sedimentary deposits affected or not by organic pollution in rivers, lakes and estuaries. Sediment assessment based on standards of organic index is shown in Table 1.

\section{Statistical analysis}

One-way ANOVA and Duncan multiple range test were used to evaluate the significant differences among different studied variables during different seasons. Duncan's multiple range tests were performed to compare the significance of means. Differences were considered significant at $\mathrm{p} \leq 0.05$.Two-way ANOVA was used for chemical parameters in order to compare sediment cores and different sites, and their interactions (Dytham, 1999).

\section{Results and discussion}

Mariout Lake has received agricultural, industrial as well as domestic Wastewater from its surrounding area. In order to study the effect of pollution on chemical properties of sediment ecosystem, sediment samples have been analyzed for chemical characteristics. The seasonal average as well as mean values of $\mathrm{pH}$ at seven sites during the period of the study is shown in Table 2. The $\mathrm{pH}$ attained its maximum value of 7.41 in winter at site (7) and its minimum of 6.81 in summer at site (3). Annual averages of $\mathrm{pH}$ varied from 6.88in site (3) to 7.24in site (6). Moreover, it increases with increasing depth.

The above results showed that the average values of $\mathrm{pH}$ were significantly $(\mathrm{p} \leq 0.05)$ increased in winter than other seasons. These finding are good in agreement with those obtained by Shaker et al., (2015), who reported that the soil $\mathrm{pH}$ depending in drainage water on flow rate from agriculture canals to lake. They added that the accumulation of organic compound were significantly $(\mathrm{p} \leq 0.05)$ increased in summer than other seasons. 
Mean concentrations of organic matter and organic carbon were significantly increased $(\mathrm{P}<0.05)$ at site 3 (18.00 and $10.46 \%$, respectively). The lowest concentrations were observed at site 4 (12.16 and 7.06\%, respectively). The seasonal average organic matter and organic carbon were preceding also normal trend as expected; it increased from (10.55 and $6.13 \%$ respectively) during winter at site 1 reaching maximum level during summer (19.99 and $11.62 \%$ respectively) in site 3 (Table 3 and 4). The organic matter and organic carbon accumulation in surface sediment were significantly increased $(\mathrm{P}<0.05)$ in summer as increasing agriculture and sewage waste water discharge from drains canals to lake. Also, the highest values of accumulation recorded in locations near the mouth of drains. These finding are good in agreement with those obtained by Shaker et al., (2014), who reported that the change in soil properties (physical and chemicals) depends on many environmental factors, including water quality, management, and the use of fertilizers and industrial feed. They added also that the organic matter and organic carbon accumulation in surface sediment in lakes depends on water quality discharge and fish wastes. Increasing the organic matter somewhere in the lake means pollution in this area with high water in its content of organic compounds and sewage waste.

Generally, the difference between sites in the accumulation of organic matter and organic carbon depends on the distance between the site and the estuaries of agricultural drains, as well as the quantities and quality of wastewater. These results are good in agreement with those obtained by El-Halag, et al., (2013).

The highest concentration of total carbon was recorded at site 3 (15.81\%) while the lowest value was recorded at sites 4 (11.62\%). In relation to the season, the minimum value of total carbon was recorded in site $7(10.30 \%)$ during winter while a maximum value was observed at site 3 (17.22\%) during summer (Table 5).

Total nitrogen and total phosphorus values were decreased at sites 2 and $1(7.20$ and $0.831 \mathrm{mg} / 100 \mathrm{~g})$, respectively while their highest values were observed at site $3(11.76$ and $1.150 \mathrm{mg} / 100 \mathrm{~g})$, respectively. The maximum value of total nitrogen was found in site 3 during summer $(13.74 \mathrm{mg} / 100 \mathrm{~g})$, whereas the minimum value was observed in site 2 during spring (5.66 $\mathrm{mg} / 100 \mathrm{~g}$ ). Total phosphorus showed maximum values in site 3 during summer $(1.478 \mathrm{mg} / 100 \mathrm{~g})$ and minimum value in site 7 during winter $(0.717 \mathrm{mg} / 100 \mathrm{~g})$ (Table 6 and 7$)$. Sediment act as a sink where phosphorus can be stored, and also as a source of phosphorus for the overlying water (Wang et al., 2014) where the ability of the sediment to absorb phosphorus 
from water depends on the texture first, as the capacity increases by increasing the clay percentage of the sediment, and environmental factors including sediment water exchange processes and depth of the overlying water (Abrams and Jarrell, 1995). We note that the sediment has a great ability to store phosphorous because of the increase in organic matter, which leads to increased absorption of phosphorous,

Two-way ANOVA (Table 8) indicated that the different sites and different cores had been affected chemical properties of sediment such as OM, OC, TC, TN, and TP. Furthermore, all the above parameter decreased with the depth. It was observed that site 3 had the higher concentration of $\mathrm{OM}, \mathrm{OC}, \mathrm{TC}, \mathrm{TN}$ and TP which indicated that this site was the most polluted site in the lake followed by site 2 . The aforementioned results might be due to increasing pollutants from Domestic and industrial wastes which discharged to the Lake without any treatment in recent years (AbdEl fattah et al., 2017).Generally, the impact of human activities in recent decades accumulated at upper layer sediment to a certain extent, subsequently resulting in accumulation of nutrients and organic matter especially in the upper layer of sediment, and their concentration decreased with the depth (Xiaojie and Cuiling, 2012).

\section{Carbon to nitrogen ratio:}

Based on sediment carbon and nitrogen ratio, the sources of nutrients can be determined to some extent. When $\omega$ (carbon) $/ \omega$ (nitrogen) $>10$, sediment organic matter is mainly exogenous organic matter, when $\omega$ (carbon) $/ \omega$ (nitrogen) $<10$, it is mainly endogenous organic matter, and when $\omega$ (carbon) $/ \omega$ (nitrogen) $=10$, it reaches balance between exogenous sources and endogenous sources (Feng $\boldsymbol{e t}$ al., 2006). The carbon nitrogen ratio of sediment in the lake ranges from 15.40 to 27.63, which indicates that sediment organic matter of the lake is mainly exogenous organic matter (Table 9).

The nitrogen ratio increases with increasing humic acid. The results clear the highest ratio of that $\mathrm{C} / \mathrm{N}$ ratio indicating the increase of industrial sewage wastewater. These results are good in agreement with those obtained by Elnady, et al., (2016).

\section{Organic Sediment Index:}

Based on the data of OSI, the sediments of Mariout Lake were characterized by a relatively low organic content (OSI values ranged from 0.41 to 0.87 ). In consequence of sediment classification concerning the standard organic indexes (Ballinger and McKee 1971), the degree of pollution at all sites was slight pollution except for sewage site which was 
considered high pollution (Table 10). The surface layer of the sediment is of great importance, whether to indicate the quality of water and fish, or the extent of the need for water quality improvement operations, or further management required as the addition of fertilizers or industrial feeds.

Table 1: Classification of sediments based in standard organic indexes (Ballinger and McKee 1971)

\begin{tabular}{|l|c|l|}
\hline Organic index & Grade & Type \\
\hline$<0.05$ & I & Clean \\
\hline$\geq 0.05-<0.20$ & II & Relative clean \\
\hline$\geq 0.20-<0.50$ & III & Slight pollution \\
\hline$\geq 0.50$ & IV & Pollution \\
\hline
\end{tabular}

Table 2: $\mathrm{pH}$ values in Lake Mariout sediment during different seasons

\begin{tabular}{|c|c|c|c|c|c|}
\hline \multirow{2}{*}{ Sites } & \multicolumn{5}{|c|}{ Depth (0-10 cm) } \\
\hline & Spring & Summer & Autumn & Winter & Mean \\
\hline 1 & $7.05^{\mathrm{bB}}$ & $7.00^{\mathrm{cB}}$ & $7.02^{\mathrm{cdB}}$ & $7.11^{\mathrm{cA}}$ & $7.04^{b}$ \\
\hline 2 & $7.22^{\mathrm{aA}}$ & $7.05^{\mathrm{bB}}$ & $7.09^{\mathrm{cB}}$ & $7.11^{\mathrm{cB}}$ & $7.11^{\mathrm{ab}}$ \\
\hline 3 & $6.85^{\mathrm{cA}}$ & $6.81^{\mathrm{cA}}$ & $6.92^{\mathrm{dA}}$ & $6.95^{\mathrm{dA}}$ & $6.88^{\mathrm{c}}$ \\
\hline 4 & $7.15^{\mathrm{bB}}$ & $7.11^{\mathrm{abB}}$ & $7.14^{\mathrm{bB}}$ & $7.24^{\mathrm{cA}}$ & $7.16^{\mathrm{ab}}$ \\
\hline 5 & $7.21^{\mathrm{aB}}$ & $7.15^{\mathrm{aB}}$ & $7.18^{\mathrm{bB}}$ & $7.33^{\mathrm{bA}}$ & $7.21^{\mathrm{a}}$ \\
\hline 6 & $7.22^{\mathrm{aB}}$ & $7.18^{\mathrm{aB}}$ & $7.22^{\mathrm{aB}}$ & $7.35^{\mathrm{bA}}$ & $7.24^{\mathrm{a}}$ \\
\hline \multirow[t]{3}{*}{7} & $7.16^{\mathrm{abB}}$ & $7.05^{\mathrm{bB}}$ & $7.11^{\mathrm{cB}}$ & $7.41^{\mathrm{aA}}$ & $7.18^{\mathrm{a}}$ \\
\hline & \multicolumn{5}{|c|}{ Depth $(10-20 \mathrm{~cm})$} \\
\hline & Spring & Summer & Autumn & Winter & Mean \\
\hline 1 & $7.25^{\mathrm{bA}}$ & $7.07^{\mathrm{cB}}$ & $7.21^{\mathrm{bB}}$ & $7.39^{\mathrm{bA}}$ & $7.23^{\mathrm{a}}$ \\
\hline 2 & $7.31^{\mathrm{aB}}$ & $7.22^{\mathrm{aC}}$ & $7.29^{\mathrm{aBC}}$ & $7.42^{\mathrm{abA}}$ & $7.31^{\mathrm{a}}$ \\
\hline 3 & $7.01^{\mathrm{cAB}}$ & $6.89^{\mathrm{dB}}$ & $6.99^{\mathrm{cB}}$ & $7.15^{\mathrm{cA}}$ & $7.01^{b}$ \\
\hline 4 & $7.23^{\mathrm{abB}}$ & $7.11^{\mathrm{bcC}}$ & $7.19^{\mathrm{bBC}}$ & $7.41^{\mathrm{abA}}$ & $7.23^{\mathrm{a}}$ \\
\hline 5 & $7.29^{\mathrm{aB}}$ & $7.17^{\mathrm{bC}}$ & $7.31^{\mathrm{aB}}$ & $7.47^{\mathrm{aA}}$ & $7.31^{\mathrm{a}}$ \\
\hline 6 & $7.31^{\mathrm{aB}}$ & $7.15^{\mathrm{bC}}$ & $7.33^{\mathrm{aB}}$ & $7.49^{\mathrm{aA}}$ & $7.32^{\mathrm{a}}$ \\
\hline 7 & $7.28^{\mathrm{aB}}$ & $7.15^{\mathrm{bC}}$ & $7.29^{\mathrm{aB}}$ & $7.42^{\mathrm{abA}}$ & $7.28^{\mathrm{a}}$ \\
\hline
\end{tabular}

Values-having different script $(a, b, c)$ at the same column are significantly $(\mathrm{P} \leq 0.05)$ different Values-having different script $(\mathrm{A}, \mathrm{B}, \mathrm{C})$ at the same row are significantly $(\mathrm{P} \leq 0.05)$ different

Site 1: Resha; Site 2: Petroget; Site 3: Sewage; Site 4: 1000 feddan; Site 5: 2000 feddan; Site 6:

3000 feddan Site 7: 6000 feddan 
Table 3: Organic matter values in Lake Mariout sediment during different s easons.

\begin{tabular}{|c|c|c|c|c|c|}
\hline \multirow{2}{*}{ Sites } & \multicolumn{5}{|c|}{ Depth (0-10 cm) } \\
\cline { 2 - 6 } & Spring & Summer & Autumn & Winter & Mean \\
\hline 1 & $12.93^{\mathrm{cB}}$ & $14.66^{\mathrm{cA}}$ & $14.02^{\mathrm{bA}}$ & $10.55^{\mathrm{dC}}$ & $13.04^{\mathrm{c}}$ \\
\hline 2 & $16.01^{\mathrm{bA}}$ & $16.35^{\mathrm{bA}}$ & $16.16^{\mathrm{aA}}$ & $14.96^{\mathrm{bB}}$ & $15.87^{\mathrm{b}}$ \\
\hline 3 & $18.56^{\mathrm{aB}}$ & $19.99^{\mathrm{aA}}$ & $16.89^{\mathrm{aC}}$ & $16.57^{\mathrm{aC}}$ & $18.00^{\mathrm{a}}$ \\
\hline 4 & $10.58^{\mathrm{fC}}$ & $13.44^{\mathrm{dA}}$ & $13.01^{\mathrm{cdA}}$ & $11.66^{\mathrm{cdB}}$ & $12.16^{\mathrm{c}}$ \\
\hline 5 & $11.12^{\mathrm{eD}}$ & $14.42^{\mathrm{cA}}$ & $13.56^{\mathrm{cB}}$ & $12.34^{\mathrm{cC}}$ & $12.86^{\mathrm{c}}$ \\
\hline 6 & $11.66^{\mathrm{dC}}$ & $13.77^{\mathrm{dA}}$ & $12.55^{\mathrm{dB}}$ & $11.03^{\mathrm{dC}}$ & $12.25^{\mathrm{c}}$ \\
\hline 7 & $11.55^{\mathrm{dB}}$ & $14.19^{\mathrm{cdA}}$ & $13.76^{\mathrm{cA}}$ & $11.02^{\mathrm{dB}}$ & $12.63^{\mathrm{c}}$ \\
\hline \multirow{2}{*}{ Sites } & & \multicolumn{5}{|c|}{ Depth $(\mathbf{1 0 - 2 0} \mathbf{c m})$} & \\
\hline & Spring & Summer & Autumn & $\mathbf{W i n t e r}^{\mathrm{ing}}$ & Mean \\
\hline & $9.32^{\mathrm{cC}}$ & $12.72^{\mathrm{cA}}$ & $11.33^{\mathrm{cB}}$ & $8.55^{\mathrm{cD}}$ & $10.48^{\mathrm{c}}$ \\
\hline 2 & $12.85^{\mathrm{bC}}$ & $14.72^{\mathrm{bA}}$ & $13.45^{\mathrm{bB}}$ & $12.11^{\mathrm{bC}}$ & $13.28^{\mathrm{b}}$ \\
\hline 3 & $15.45^{\mathrm{aB}}$ & $16.99^{\mathrm{aA}}$ & $15.35^{\mathrm{aB}}$ & $14.32^{\mathrm{aC}}$ & $15.52^{\mathrm{a}}$ \\
\hline 4 & $8.35^{\mathrm{dC}}$ & $10.54^{\mathrm{eA}}$ & $9.11^{\mathrm{eB}}$ & $8.77^{\mathrm{cC}}$ & $9.19^{\mathrm{c}}$ \\
\hline 5 & $9.55^{\mathrm{cB}}$ & $11.57^{\mathrm{dA}}$ & $10.66^{\mathrm{dA}}$ & $8.84^{\mathrm{cC}}$ & $10.15^{\mathrm{c}}$ \\
\hline 6 & $9.47^{\mathrm{cC}}$ & $10.88^{\mathrm{eA}}$ & $10.01^{\mathrm{dB}}$ & $7.12^{\mathrm{dD}}$ & $9.37^{\mathrm{c}}$ \\
\hline 7 & $9.35^{\mathrm{cB}}$ & $11.14^{\mathrm{dA}}$ & $9.75^{\mathrm{eB}}$ & $7.67^{\mathrm{dC}}$ & $9.47^{\mathrm{c}}$ \\
\hline
\end{tabular}

Values-having different script $(a, b, c)$ at the same column are significantly $(\mathrm{P} \leq 0.05)$ different

Values-having different script $(\mathrm{A}, \mathrm{B}, \mathrm{C})$ at the same row are significantly $(\mathrm{P} \leq 0.05)$ different

Site 1: Resha; Site 2: Petroget; Site 3: Sewage; Site 4: 1000 feddan; Site 5: 2000 feddan; Site 6: 3000 feddan Site 7: 6000 feddan 
Tabl e 4: Organic carbon values in Lake Mariout sediment during different seasons.

\begin{tabular}{|c|c|c|c|c|c|}
\hline \multirow{2}{*}{ Sites } & \multicolumn{5}{|c|}{ Depth $(0-10 \mathrm{~cm})$} \\
\hline & Spring & Summer & Autumn & Winter & Mean \\
\hline 1 & $7.51^{\mathrm{cB}}$ & $8.52^{\mathrm{cA}}$ & $8.15^{\mathrm{bA}}$ & $6.13^{\mathrm{dC}}$ & $7.57^{\mathrm{c}}$ \\
\hline 2 & $9.30^{\mathrm{bA}}$ & $9.50^{\mathrm{bA}}$ & $9.39^{\mathrm{aA}}$ & $8.69^{\mathrm{bB}}$ & $9.22^{b}$ \\
\hline 3 & $10.79^{\mathrm{aB}}$ & $11.62^{\mathrm{aA}}$ & $9.81^{\mathrm{aC}}$ & $9.63^{\mathrm{aC}}$ & $10.46^{\mathrm{a}}$ \\
\hline 4 & $6.14^{\mathrm{eB}}$ & $7.81^{\mathrm{eA}}$ & $7.56^{\mathrm{cA}}$ & $6.77^{\mathrm{cdB}}$ & $7.06^{\mathrm{c}}$ \\
\hline 5 & $6.46^{\mathrm{deC}}$ & $8.38^{\mathrm{cA}}$ & $7.88^{\mathrm{cB}}$ & $7.17^{\mathrm{cB}}$ & $7.47^{\mathrm{c}}$ \\
\hline 6 & $6.77^{\mathrm{dC}}$ & $8.00^{\mathrm{dA}}$ & $7.29^{\mathrm{dB}}$ & $6.41^{\mathrm{cdC}}$ & $7.11^{\mathrm{c}}$ \\
\hline \multirow[t]{3}{*}{7} & $6.71^{\mathrm{dC}}$ & $8.25^{\mathrm{cA}}$ & $8.00^{\mathrm{bB}}$ & $6.40^{\mathrm{cdC}}$ & $7.34^{\mathrm{c}}$ \\
\hline & \multicolumn{5}{|c|}{ Depth $(10-20 \mathrm{~cm})$} \\
\hline & Spring & Summer & Autumn & Winter & Mean \\
\hline 1 & $5.41^{\mathrm{cC}}$ & $7.39^{\mathrm{cA}}$ & $6.58^{\mathrm{cB}}$ & $4.97^{\mathrm{cD}}$ & $6.08^{c}$ \\
\hline 2 & $7.47^{\mathrm{bC}}$ & $8.55^{\mathrm{bA}}$ & $7.81^{\mathrm{bB}}$ & $7.04^{\mathrm{bD}}$ & $7.71^{b}$ \\
\hline 3 & $8.98^{\mathrm{aB}}$ & $9.87^{\mathrm{aA}}$ & $8.92^{\mathrm{aB}}$ & $8.32^{\mathrm{aB}}$ & $9.02^{\mathrm{a}}$ \\
\hline 4 & $4.85^{\mathrm{dC}}$ & $6.12^{\mathrm{eA}}$ & $5.29^{\mathrm{eB}}$ & $5.09^{\mathrm{cB}}$ & $5.33^{\mathrm{c}}$ \\
\hline 5 & $5.55^{\mathrm{cB}}$ & $6.72^{\mathrm{dA}}$ & $6.19^{\mathrm{cA}}$ & $5.13^{\mathrm{cB}}$ & $5.89^{c}$ \\
\hline 6 & $5.50^{\mathrm{cB}}$ & $6.32^{\mathrm{dA}}$ & $5.81^{\mathrm{dB}}$ & $4.13^{\mathrm{dC}}$ & $5.44^{\mathrm{c}}$ \\
\hline 7 & $5.43^{\mathrm{cB}}$ & $6.47^{\mathrm{dA}}$ & $5.66^{\mathrm{dB}}$ & $4.45^{\mathrm{dC}}$ & $5.50^{c}$ \\
\hline
\end{tabular}

Values-having different script $(\mathrm{a}, \mathrm{b}, \mathrm{c})$ at the same column are significantly $(\mathrm{P} \leq 0.05)$ different Values-having different script $(\mathrm{A}, \mathrm{B}, \mathrm{C})$ at the same row are significantly $(\mathrm{P} \leq 0.05)$ different

Site 1: Resha; Site 2: Petroget; Site 3: Sewage; Site 4: 1000 feddan; Site 5: 2000 feddan; Site 6: 3000 feddan Site 7: 6000 feddan 
Tale 5: T otal carbon values in Lake Mariout sediment during different seasons.

\begin{tabular}{|c|c|c|c|c|c|}
\hline \multirow{2}{*}{ Sites } & \multicolumn{5}{|c|}{ Depth $(0-10 \mathrm{~cm})$} \\
\hline & Spring & Summer & Autumn & Winter & Mean \\
\hline 1 & $12.81^{\mathrm{cC}}$ & $14.12^{\mathrm{cA}}$ & $13.55^{\mathrm{bB}}$ & $11.13^{\mathrm{cD}}$ & $12.90^{\mathrm{bc}}$ \\
\hline 2 & $14.90^{\mathrm{bB}}$ & $15.29^{\mathrm{bA}}$ & $15.20^{\mathrm{aA}}$ & $14.09^{\mathrm{aB}}$ & $14.87^{\mathrm{ab}}$ \\
\hline 3 & $16.09^{\mathrm{aB}}$ & $17.22^{\mathrm{aA}}$ & $15.31^{\mathrm{aC}}$ & $14.63^{\mathrm{aD}}$ & $15.81^{\mathrm{a}}$ \\
\hline 4 & $11.53^{\mathrm{dB}}$ & $11.51^{\mathrm{eB}}$ & $12.96^{\mathrm{bA}}$ & $11.57^{\mathrm{bB}}$ & $11.89^{c}$ \\
\hline 5 & $12.17^{\mathrm{cB}}$ & $13.10^{\mathrm{cA}}$ & $12.89^{\mathrm{bB}}$ & $11.81^{\mathrm{bC}}$ & $12.49^{c}$ \\
\hline 6 & $10.66^{\mathrm{eC}}$ & $12.38^{\mathrm{dA}}$ & $12.08^{\mathrm{cA}}$ & $11.37^{\mathrm{bB}}$ & $11.62^{\mathrm{c}}$ \\
\hline \multirow[t]{3}{*}{7} & $11.51^{\mathrm{dC}}$ & $13.55^{\mathrm{cA}}$ & $12.30^{\mathrm{cB}}$ & $10.30^{\mathrm{cD}}$ & $11.92^{\mathrm{c}}$ \\
\hline & \multicolumn{5}{|c|}{ Depth $(10-20 \mathrm{~cm})$} \\
\hline & Spring & Summer & Autumn & Winter & Mean \\
\hline 1 & $10.61^{\mathrm{bC}}$ & $12.99^{\mathrm{cA}}$ & $11.78^{\mathrm{bB}}$ & $8.87^{\mathrm{cD}}$ & $11.06^{\mathrm{bc}}$ \\
\hline 2 & $13.17^{\mathrm{aB}}$ & $14.05^{\mathrm{bA}}$ & $12.31^{\mathrm{aC}}$ & $11.24^{\mathrm{aD}}$ & $12.69^{\mathrm{ab}}$ \\
\hline 3 & $13.68^{\mathrm{aB}}$ & $15.17^{\mathrm{aA}}$ & $13.92^{\mathrm{aB}}$ & $12.82^{\mathrm{aC}}$ & $13.89^{\mathrm{a}}$ \\
\hline 4 & $7.25^{\mathrm{dC}}$ & $9.39^{\mathrm{eA}}$ & $8.92^{\mathrm{eB}}$ & $8.79^{\mathrm{cB}}$ & $8.58^{\mathrm{e}}$ \\
\hline 5 & $9.65^{\mathrm{cB}}$ & $10.42^{\mathrm{dA}}$ & $10.09^{\mathrm{cA}}$ & $9.03^{\mathrm{bB}}$ & $9.79^{\mathrm{cd}}$ \\
\hline 6 & $10.00^{\mathrm{bB}}$ & $11.37^{\mathrm{dA}}$ & $11.31^{\mathrm{bA}}$ & $9.43^{\mathrm{bB}}$ & $10.51^{\mathrm{cd}}$ \\
\hline 7 & $7.93^{\mathrm{dB}}$ & $9.56^{\mathrm{eA}}$ & $9.37^{\mathrm{dA}}$ & $8.05^{\mathrm{dB}}$ & $8.72^{\mathrm{de}}$ \\
\hline
\end{tabular}

Values-having different script $(\mathrm{a}, \mathrm{b}, \mathrm{c})$ at the same column are significantly $(\mathrm{P} \leq 0.05)$ different

Values-having different script $(\mathrm{A}, \mathrm{B}, \mathrm{C})$ at the same row are significantly $(\mathrm{P} \leq 0.05)$ different

Site 1: Resha; Site 2: Petroget; Site 3: Sewage; Site 4: 1000 feddan; Site 5: 2000 feddan; Site 6: 3000 feddan Site 7: 6000 feddan 
Table 6 : Average values of total nitrogen as $\mathrm{mg} / 100 \mathrm{~g}$ sediment in Lake Mariout during different seasons.

\begin{tabular}{|c|c|c|c|c|c|}
\hline \multirow{2}{*}{ Sites } & \multicolumn{5}{|c|}{ Depth (0-10 cm) } \\
\cline { 2 - 6 } & Spring & Summer & Autumn & Winter & Mean \\
\hline 1 & $7.22^{\mathrm{bB}}$ & $8.88^{\mathrm{cA}}$ & $8.11^{\mathrm{dA}}$ & $7.55^{\mathrm{cB}}$ & $7.94^{\mathrm{b}}$ \\
\hline 2 & $5.66^{\mathrm{dC}}$ & $7.97^{\mathrm{dA}}$ & $8.07^{\mathrm{dA}}$ & $7.11^{\mathrm{dB}}$ & $7.20^{\mathrm{b}}$ \\
\hline 3 & $9.12^{\mathrm{aD}}$ & $13.74^{\mathrm{aA}}$ & $12.55^{\mathrm{aB}}$ & $11.66^{\mathrm{aC}}$ & $11.76^{\mathrm{a}}$ \\
\hline 4 & $7.55^{\mathrm{cC}}$ & $9.07^{\mathrm{cA}}$ & $8.84^{\mathrm{cB}}$ & $8.03^{\mathrm{bB}}$ & $8.37^{\mathrm{b}}$ \\
\hline 5 & $7.05^{\mathrm{cB}}$ & $8.66^{\mathrm{A}}$ & $8.44^{\mathrm{cA}}$ & $7.12^{\mathrm{dB}}$ & $7.81^{\mathrm{b}}$ \\
\hline 6 & $7.66^{\mathrm{cB}}$ & $9.18^{\mathrm{bcA}}$ & $9.11^{\mathrm{bA}}$ & $7.45^{\mathrm{cB}}$ & $8.35^{\mathrm{b}}$ \\
\hline 7 & $8.11^{\mathrm{bB}}$ & $9.77^{\mathrm{bA}}$ & $9.45^{\mathrm{bA}}$ & $7.94^{\mathrm{bcB}}$ & $8.81^{\mathrm{b}}$ \\
\hline Sites & \multicolumn{5}{|c|}{ Depth $(\mathbf{1 0 - 2 0} \mathbf{c m})$} \\
\hline & Spring & Summer & Autumn & Winter & Mean \\
\hline & $5.75^{\mathrm{bC}}$ & $7.11^{\mathrm{cA}}$ & $6.63^{\mathrm{bB}}$ & $5.54^{\mathrm{bC}}$ & $6.25^{\mathrm{a}}$ \\
\hline 2 & $3.76^{\mathrm{dC}}$ & $5.07^{\mathrm{eA}}$ & $4.72^{\mathrm{dB}}$ & $3.44^{\mathrm{dC}}$ & $4.24^{\mathrm{b}}$ \\
\hline 3 & $6.66^{\mathrm{aC}}$ & $8.15^{\mathrm{aA}}$ & $7.87^{\mathrm{aB}}$ & $6.36^{\mathrm{aC}}$ & $7.26^{\mathrm{a}}$ \\
\hline 4 & $5.21^{\mathrm{bC}}$ & $7.05^{\mathrm{cdA}}$ & $6.47^{\mathrm{bB}}$ & $4.86^{\mathrm{CD}}$ & $5.89^{\mathrm{a}}$ \\
\hline 5 & $4.88^{\mathrm{cB}}$ & $6.87^{\mathrm{dA}}$ & $6.22^{\mathrm{cA}}$ & $5.11^{\mathrm{bB}}$ & $5.77^{\mathrm{a}}$ \\
\hline 6 & $4.65^{\mathrm{cD}}$ & $7.14^{\mathrm{c}}$ & $6.48^{\mathrm{bB}}$ & $5.03^{\mathrm{bC}}$ & $5.82^{\mathrm{a}}$ \\
\hline 7 & $5.51^{\mathrm{bC}}$ & $7.55^{\mathrm{bA}}$ & $7.22^{\mathrm{aA}}$ & $6.33^{\mathrm{aB}}$ & $6.65^{\mathrm{a}}$ \\
\hline
\end{tabular}

Values-having different script $(\mathrm{a}, \mathrm{b}, \mathrm{c})$ at the same column are significantly $(\mathrm{P} \leq 0.05)$ different

Values-having different script $(\mathrm{A}, \mathrm{B}, \mathrm{C})$ at the same row are significantly $(\mathrm{P} \leq 0.05)$ different

Site 1: Resha; Site 2: Petroget; Site 3: Sewage; Site 4: 1000 feddan; Site 5: 2000 feddan; Site 6: 3000 feddan Site 7: 6000 feddan 
Table 7: Average values of total phosphorus as mg/100g sediment in Lake Mariout during different seasons.

\begin{tabular}{|c|c|c|c|c|c|}
\hline \multirow{2}{*}{ Sites } & \multicolumn{5}{|c|}{ Depth $(0-10 \mathrm{~cm})$} \\
\hline & Spring & Summer & Autumn & Winter & Mean \\
\hline 1 & $0.758^{\mathrm{dC}}$ & $0.987^{\mathrm{cA}}$ & $0.813^{\mathrm{cB}}$ & $0.768^{\mathrm{cC}}$ & $0.831^{\mathrm{b}}$ \\
\hline 2 & $0.987^{\mathrm{bB}}$ & $1.121^{\mathrm{bA}}$ & $0.978^{\mathrm{bB}}$ & $0.797^{\mathrm{cC}}$ & $0.970^{\mathrm{ab}}$ \\
\hline 3 & $1.014^{\mathrm{aB}}$ & $1.478^{\mathrm{aA}}$ & $1.122^{\mathrm{aA}}$ & $0.989^{\mathrm{aC}}$ & $1.150^{\mathrm{a}}$ \\
\hline 4 & $0.894^{\mathrm{cB}}$ & $1.022^{\mathrm{bA}}$ & $1.007^{\mathrm{aA}}$ & $0.842^{\mathrm{bB}}$ & $0.941^{\mathrm{ab}}$ \\
\hline 5 & $0.898^{\mathrm{cC}}$ & $1.127^{\mathrm{bA}}$ & $0.954^{\mathrm{bB}}$ & $0.762^{\mathrm{cD}}$ & $0.935^{\mathrm{ab}}$ \\
\hline 6 & $0.954^{\mathrm{bB}}$ & $1.117^{\mathrm{bA}}$ & $1.123^{\mathrm{aA}}$ & $0.889^{\mathrm{bC}}$ & $1.020^{\mathrm{ab}}$ \\
\hline \multirow[t]{3}{*}{7} & $0.782^{\mathrm{dC}}$ & $0.935^{\mathrm{cA}}$ & $0.894^{\mathrm{cB}}$ & $0.717^{\mathrm{dC}}$ & $0.832^{\mathrm{b}}$ \\
\hline & \multicolumn{5}{|c|}{ Depth $(10-20 \mathrm{~cm})$} \\
\hline & Spring & Summer & Autumn & Winter & Mean \\
\hline 1 & $0.588^{\mathrm{dB}}$ & $0.777^{\mathrm{cA}}$ & $0.747^{\mathrm{cA}}$ & $0.445^{\mathrm{cC}}$ & $0.639^{b}$ \\
\hline 2 & $0.714^{\mathrm{bD}}$ & $0.827^{\mathrm{bA}}$ & $0.795^{\mathrm{cB}}$ & $0.519^{\mathrm{bC}}$ & $0.713^{\mathrm{ab}}$ \\
\hline 3 & $0.857^{\mathrm{aC}}$ & $0.976^{\mathrm{aA}}$ & $0.905^{\mathrm{aB}}$ & $0.723^{\mathrm{aD}}$ & $0.865^{\mathrm{a}}$ \\
\hline 4 & $0.522^{\mathrm{dB}}$ & $0.685^{\mathrm{dA}}$ & $0.654^{\mathrm{dA}}$ & $0.507^{\mathrm{bB}}$ & $0.592^{b}$ \\
\hline 5 & $0.627^{\mathrm{cB}}$ & $0.842^{\mathrm{bA}}$ & $0.811^{\mathrm{bA}}$ & $0.527^{\mathrm{bC}}$ & $0.701^{\mathrm{ab}}$ \\
\hline 6 & $0.517^{\mathrm{dB}}$ & $0.734^{\mathrm{cA}}$ & $0.617^{\mathrm{eB}}$ & $0.408^{\mathrm{cD}}$ & $0.569^{\mathrm{b}}$ \\
\hline 7 & $0.543^{\mathrm{dB}}$ & $0.708^{\mathrm{cA}}$ & $0.698^{\mathrm{dB}}$ & $0.512^{\mathrm{bB}}$ & $0.615^{\mathrm{b}}$ \\
\hline
\end{tabular}

Values-having different script $(\mathrm{a}, \mathrm{b}, \mathrm{c})$ at the same column are significantly $(\mathrm{P} \leq 0.05)$ different

Values-having different script $(\mathrm{A}, \mathrm{B}, \mathrm{C})$ at the same row are significantly $(\mathrm{P} \leq 0.05)$ different

Site 1: Resha; Site 2: Petroget; Site 3: Sewage; Site 4: 1000 feddan; Site 5: 2000 feddan; Site 6: 3000 feddan Site 7: 6000 feddan 
Table 8: Two-Way ANOVA and main effect of sites and core on chemical properties in Mariout Lake sediments.

\begin{tabular}{|c|c|c|c|c|c|c|c|}
\hline & & pH & $\mathbf{O M}$ & $\mathrm{OC}$ & $\mathrm{TC}$ & $\mathbf{T N}$ & $\mathbf{T P}$ \\
\hline \multirow{3}{*}{$\begin{array}{l}\text { Source of } \\
\text { variation }\end{array}$} & Sites & $* *$ & $* *$ & $*$ & $* *$ & $* *$ & $* *$ \\
\hline & Core & $* *$ & $* *$ & $*$ & $*$ & $*$ & $* *$ \\
\hline & $\begin{array}{c}\text { Sites and } \\
\text { core }\end{array}$ & ns & ns & ns & $*$ & $*$ & $*$ \\
\hline \multirow{7}{*}{ Sites } & 1 & $7.13^{b}$ & $11.76^{\mathrm{c}}$ & $6.83^{c}$ & $11.98^{\mathrm{c}}$ & $7.09^{b}$ & $0.735^{\mathrm{b}}$ \\
\hline & 2 & $7.22^{\mathrm{ab}}$ & $14.57^{b}$ & $8.47^{b}$ & $13.78^{b}$ & $5.72^{c}$ & $0.842^{b}$ \\
\hline & 3 & $6.95^{c}$ & $16.76^{\mathrm{a}}$ & $9.74^{\mathrm{a}}$ & $14.86^{\mathrm{a}}$ & $9.51^{\mathrm{a}}$ & $1.008^{\mathrm{a}}$ \\
\hline & 4 & $7.19^{\mathrm{ab}}$ & $10.67^{c}$ & $6.20^{c}$ & $10.24^{\mathrm{e}}$ & $7.13^{b}$ & $0.767^{b}$ \\
\hline & 5 & $7.26^{\mathrm{a}}$ & $11.57^{\mathrm{c}}$ & $6.73^{c}$ & $10.71^{\mathrm{de}}$ & $6.79^{b}$ & $0.818^{b}$ \\
\hline & 6 & $7.28^{\mathrm{a}}$ & $10.79^{c}$ & $6.27^{c}$ & $11.51^{\mathrm{cd}}$ & $7.08^{b}$ & $0.795^{b}$ \\
\hline & 7 & $7.23^{\mathrm{ab}}$ & $11.05^{\mathrm{c}}$ & $6.42^{c}$ & $10.32^{\mathrm{e}}$ & $7.78^{b}$ & $0.743^{b}$ \\
\hline \multirow{2}{*}{ Cores } & Surface & $7.12^{b}$ & $13.84^{\mathrm{a}}$ & $8.05^{\mathrm{a}}$ & $13.07^{\mathrm{a}}$ & $8.61^{\mathrm{a}}$ & $0.960^{\mathrm{a}}$ \\
\hline & Subsurface & $7.25^{\mathrm{a}}$ & $11.07^{b}$ & $6.43^{b}$ & $10.75^{b}$ & $6.00^{b}$ & $0.671^{b}$ \\
\hline
\end{tabular}

Sign. $=$ significance level $*=$ significant $(\mathrm{P} \leq 0.05),{ }^{* *}=$ highly significant $(\mathrm{P} \leq 0.01)$, and $\mathrm{ns}=$ not significant 
Table 9: Carbon to nitrogen ratio of sediment samples from Mariout Lake.

\begin{tabular}{|c|c|c|c|}
\hline Sites & $\mathbf{C ~ \%}$ & $\mathbf{N \%}$ & $\mathbf{C} / \mathbf{N}$ \\
\hline 1 & 0.981 & 0.054 & 17.97 \\
\hline 2 & 1.217 & 0.044 & 27.63 \\
\hline 3 & 1.399 & 0.073 & 19.11 \\
\hline 4 & 0.890 & 0.054 & 16.22 \\
\hline 5 & 0.967 & 0.052 & 18.49 \\
\hline 6 & 0.900 & 0.054 & 16.52 \\
\hline 7 & 0.922 & 0.059 & 15.40 \\
\hline
\end{tabular}

Table 10: OSI values of sediment samples from Mariout Lake.

\begin{tabular}{|c|c|c|c|}
\hline Sites & OC wt\% & ON wt\% & OSI \\
\hline 1 & 6.82 & 0.067 & 0.46 \\
\hline 2 & 8.46 & 0.054 & 0.45 \\
\hline 3 & 9.74 & 0.090 & 0.87 \\
\hline 4 & 6.19 & 0.067 & 0.41 \\
\hline 5 & 6.68 & 0.064 & 0.43 \\
\hline 6 & 6.27 & 0.067 & 0.42 \\
\hline 7 & 6.42 & 0.073 & 0.47 \\
\hline
\end{tabular}

\section{Conclusion}

The present data indicated that

1- In general, the chemical parameters of lake sediment including, organic matter, organic carbon, total carbon, total nitrogen and total phosphorus are concentrated in surface sediment and decrease with the increase of the depth.

2- Concerning the degree of sediment pollution, the majority of studied areas in Mariout Lake considered of slight pollution except for sewage site which was highly polluted by organic matter and the sediment should be cleared.

3- The organic matter of sediment in Mariout Lake is mainly of exogenous organic matter. Thus the control of sediment nutrient concentrations depends upon the control of exogenous pollution. 


\section{References}

Abrams, M.M. and Jarrell, W.M. (1995): soil phosphorus as a potential non-point source for elevated stream phosphorus levels. J. Ennviron qual. 24: 132-138.

A.O.A.C. Association of Official Agriculture Chemists (1990): Official Methods of Analysis of the Association of Official Analytical Chemists, $15^{\text {th }}$ ed. Washington, D.C.

A.P.H.A. American Public Health Association (1985): Standard Methods for the Examination of Water and Wastewater. $16^{\text {th }}$ ed., Washington, D.C., p1268.

Ballinger, D.G. and Mckee, G.D. (1971): chemical characterization of bottom sediments. J. Water pollution control federation, 43(2):216-227.

Dytham, C. (1999): Choosing and using statistics: A Biologist's guide. Blackwell science Ltd., London, UK.

Elnady, A.M, Shaker, I.M, Abdel Wahed, R.K, Soliman, M.A.M (2016): Impact of Fish Farm Management on Physico-chemical Properties of Water and Sediments in earthen ponds.Egy. J. Aquac., Vol. 6, No. (2):120. ISSN: 2090-7877.

El-Halag, R.S.F., Shaker, I.M., Sahar F. M; Mohammed. F. O and Alam ,E.F. (2013): Impact of Some Environmental Condition on Water Quality and Some Heavy metals in Water FromBardawil Lake. New York Science Journal; 6(11).

Feng F, Wang H, Fang T, Liu JT (2006): The correlation between microbial biomass and carbon, nitrogen, phosphorus in thesediments of Lake Donghu. China Environmental Science., 26: 342-345.

Gehan M. El Zokm, Hermine R.Z. Tadros, Mohamed A. Okbah and Galila H. Ibrahim (2018): Eutrophication assessment using TRIX and Carlson's indices in Lake Mariout Water, Egypt. Egyptian Journal of Aquatic Biology and Fisheries Vol. 22(5): 321- 339

Kucuksezgin, F., Kontas, A., Altay, O., Uluturhan, E., and Darnlmaz E. (2006): Assessment of marine pollution in Izmir Bay: Nutrient, heavy metal and total hydrocarbon concentrations. Environ. Int., 32: 41-51

Leoni, L, and Sortori, F.: (1996): Heavy metals and arsenic in sediments from continental shelf of the Northern Tyrrhenian/Eastern Ligurian seas. Mar. Environ. Res, 41(1): 73-98.

Mateo M. A. (2009): Lake Mariut: An ecological assessment. WADI project water demand integration; INCO-CT-2005-015226. 
Saad, A.S.; Massoud, R.A. and Ghorab, M.A. (2017): Assessment of the Physicochemical Characteristics and Water Quality Analysis of Mariout Lake, Southern of Alexandria, Egypt. J Environ Anal Toxicology, 7(1): 1-19.

Scapini, F. (2009): Sustainable management of Mediterranean coastal fresh and transitional water bodies: a socio-economic and environmental analysis of changes and trends to enhance and sustain stakeholder benefits. Coastal Water Bodies Nature and Culture Conflicts in the Mediterranean 1-5.

Shaker, I.M.;El-Dahhar, A.A. and Darwish, S. (2015): Impacts of Some Environmental Condition on Water Quality and Some Heavy Metals in Water from Burullus Lake. J Arabian Aquaculture Society. Arabian Aquaculture Conference. Vol. 10 No 2, 155-172.

Shaker, I.M., Rabie S. F. El-Halag, R.S.F. Sahar F. M; Mohammed, F. O and Alam, E. F. (2014): Assessment of Physical, Chemical Properties and Some Heavy Metals in Sediment of Bardawil Lake, Egypt. Impress in Abbassa, Int., J. Aqua.

Tavares S. and Boyd, C. (2003): Comparison of a dry ash method with perchloric acid digestion for total phosphorus analysis of pond sediment. J. Aquaculture in the Tropics, 18: 239-244.

Wang, L., Liang, T., Zhong, B., Lik, Z. Q. (2014): Study on phosphorus dynamics at the sediment-water interface of Dongting lake, China. Aquat Geochem. 20 (5): 1-17.

Xiaojie, D. and Cuiling, J. (2012): Pollution characteristics of nutrients in sediment at changtan reservoir. Procedia engineering, 28: 178-181. 


\section{تأثير الملوثات علي بعض الخصائص الكيمائية لراسب بحيرة مصر مريوط

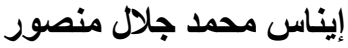 \\ قسم الليمنولوجي, المعمل المركزي لبحوث الثروة السمكية, العباسة, ابو حماد, شرقية الملخص العربي}

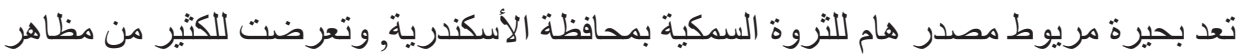

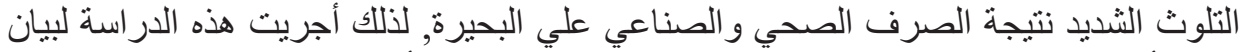

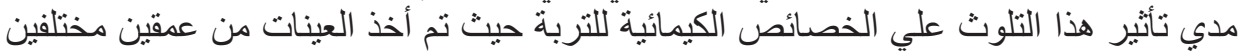

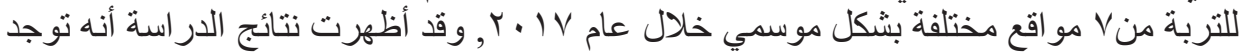

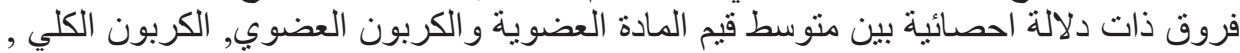

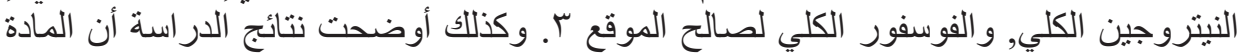

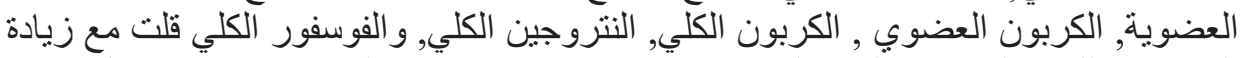

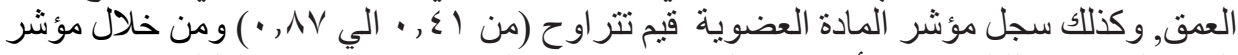

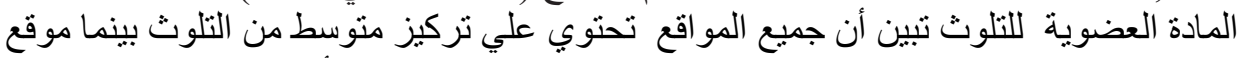

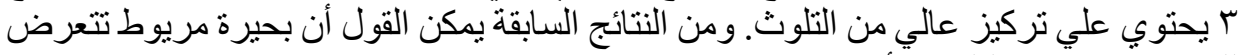

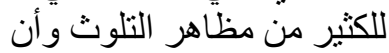
هذا التلوث مصدره خارجي, لذلك من الممكن التحكم في مصادر التلوث الذي تتعرض له البحيرة. 